\title{
Contributions of Intrinsic Renal Cells to Crescentic Glomerulonephritis
}

\author{
Peter G. Tipping Jennifer Timoshanko \\ Centre for Inflammatory Diseases, Department of Medicine, Monash University, Clayton, Australia
}

\author{
Key Words \\ Macrophage $\cdot$ T cell $\cdot$ Cytokine $\cdot$ Cell-mediated \\ immunity
}

\begin{abstract}
The pro-inflammatory contributions of leukocytes, particularly macrophages and T cells, to the immunopathogenesis of proliferative forms of glomerulonephritis (GN) have been clearly established by various techniques, including in vivo depletion studies in experimental models. The evidence for an active pro-inflammatory role for intrinsic renal cells in GN has relied on studies demonstrating their production of pro-inflammatory mediators in vitro and during the development of GN. Until recently, the specific in vivo contributions of mediators from intrinsic renal cells to inflammatory injury in GN have proven difficult to define. Utilising 'chimeric' mice as a tool, several studies have explored the involvement of intrinsic renal cells via their production of cytokines and other key pro-inflammatory molecules. These studies provide evidence of important functional contributions of intrinsic renal cells to inflammatory injury in $\mathrm{GN}$ via their expression of cytokines, cytokine receptors, MHC-II and co-stimulatory molecules. They suggest a sequence of interactions between cytokines from leuko-
\end{abstract}

cytes and intrinsic renal cells and important contributions of glomerular epithelial cell proliferation to crescent formation.

Copyright $(2005$ S. Karger AG, Basel

\section{Introduction}

Proliferative and particularly crescentic forms of glomerulonephritis $(\mathrm{GN})$ are associated with prominent glomerular accumulation of leukocytes and severe renal injury. Macrophages, because of their abundance (particularly in crescentic human glomeruli), their association with features of severe glomerular injury and their wide range of pro-inflammatory capacities, have been considered to be the major cellular mediator of injury in proliferative and crescentic forms of GN. The presence of $\mathrm{CD}^{+} \mathrm{T}$ cells, macrophages and fibrin in crescentic GN indicates involvement of 'delayed-type hypersensitivity'like cell-mediated immunity in the development of glomerular injury.

Intrinsic renal cells have been shown to acquire proinflammatory phenotypes in vitro. Mesangial cells and glomerular epithelial cells produce a variety of pro-inflammatory mediators in vitro, including cytokines, chemokines, reactive oxygen metabolites, nitric oxide,

\section{KARGER \\ Fax +4161306 1234 E-Mail karger@karger.ch} www.karger.com
(C) 2005 S. Karger AG, Basel 1660-2129/05/1014-0173\$22.00/0

Accessible online at:

www.karger.com/nee
Prof. P.G. Tipping

Centre for Inflammatory Diseases

Department of Medicine, Monash University

Clayton, Vic. 3800 (Australia)

Tel. +6139594 5547, Fax +6139594 6495, E-Mail peter.tipping@med.monash.edu.au 
complement components and prostanoids. Immunohistochemical studies and in situ hybridization have demonstrated the presence of various cytokines and/or their mRNA in human and experimental GN. Functional evidence for their contribution to inflammatory injury in GN has been provided by in vivo inhibition studies and the use of cytokine-deficient mice in experimental models. However, demonstration of the extent to which the pro-inflammatory functions of intrinsic renal cells contribute to glomerular injury in GN has required application of newer techniques in experimental models.

\section{Bone Marrow Transplantation and Generation of Chimeric Mice}

The use of bone marrow transplantation in inbred strains of mice has recently proved a useful tool to address the relative contributions of intrinsic renal cells and bone marrow-derived leukocytes in a number of in vivo models of inflammatory injury. Transplantation of bone marrow in highly inbred strains following lethal irradiation allows almost complete replacement of the recipient's marrow and circulating leukocytes with cells from a donor with specific genetic alteration. This technique creates 'chimeric' mice in which bone marrow-derived leukocytes can express different inflammatory phenotypes to non-bone marrow-derived tissues such as the kidney. Circulating leukocytes and other haematological parameters are restored to normal values within 8 weeks, typically with $95 \%$ of the reconstituted $\mathrm{CD} 45^{+}$leukocytes showing the donor phenotype [1].

Although the potential for bone marrow-derived stem cells to contribute to resident parenchymal cells in various organs including the kidney has been demonstrated, bone marrow-derived cells make up only a small faction of the resident renal cells at least in the first 2-3 months following transplantation $[2,3]$. Transplantation of bone marrow from a donor with a single cytokine deficiency generates a 'chimeric' mouse in which the vast majority of leukocytes are unable to produce the cytokine, whilst non-bone marrow-derived cells are normal. Conversely, transplantation of normal marrow into a cytokine-deficient mouse results in selective restoration of the capacity to produce that cytokine in bone marrow-derived cells alone. These chimeric mice allow dissection of functional contributions of leukocytes and intrinsic renal cells in crescentic GN, with regard to their capacity to contribute to injury via specific cytokines, antigen recognition and co-stimulation and their proliferative capacity.

\section{Cytokine Production by Intrinsic Renal Cells}

TNF and $I L-1 \beta$

Both TNF and IL-1 $\beta$ are important mediators of injury in GN. TNF is produced by leukocytes, including monocytes/macrophages, activated $\mathrm{CD} 4^{+} \mathrm{T}$ cells, natural killer cells and mast cells and by intrinsic renal cells, including mesangial cells and tubular epithelial cells. IL-1 is also produced by macrophages and mesangial cells. TNF expression is prominent in crescents, infiltrating macrophages and tubular epithelial cells in patients with ANCA-associated GN (particularly those with active glomerular lesions) [4] and also in membranous GN [5]. IL-1 expression has been shown in glomeruli in crescentic forms of human [4] and experimental GN [6]. An important contribution of these cytokine to development of experimental GN has been demonstrated by attenuation of disease in TNF- and IL-1 $\beta$-deficient mice and by in vivo inhibition by administration of soluble TNF receptors, IL-1 receptor antagonist (IL-1 ra), anti-TNF or anti-IL-1 antibodies. Transplantation of bone marrow over expressing IL-1 ra can also suppress development of antiGBM GN in mice [7].

In crescentic $\mathrm{GN}$ in rabbits, glomerular macrophages have been shown to be an abundant source of TNF [8] and IL-1 [9], but in rats, immunohistological studies have suggested that intrinsic cells are the major source of IL-1 $\beta$ [6]. The use of TNF and IL-1 $\beta$ chimeric mice has allowed in vivo comparison of the relative functional contributions of cytokine production by intrinsic renal cells and leukocytes to immune renal injury. These studies show contrasting contributions of leukocytes and intrinsic renal cells to crescentic glomerular injury mediated by these two cytokines.

In a mouse model of crescentic GN induced by administration of sheep anti GBM antibody, glomerular injury and crescent formation was substantially dependent on intrinsic renal cell production of TNF. Crescent formation, functional glomerular injury, renal expression of MHC-II (particularly by tubular cells), E-selectin expression on endothelial cells and glomerular fibrin deposition were all significantly inhibited in mice lacking the capacity for TNF production by renal cells [10]. The extent of protection was similar to that seen in mice with total TNF deficiency. In contrast, chimeric mice with TNF-deficient leukocytes but intact intrinsic renal cell-derived TNF developed severe proliferative GN similar to wild-type mice. Studies of chimeric TNFR2-deficient mice showed expression of this TNF receptor on intrinsic renal cells played a dominant role over leukocyte expression in 
the development of anti-GBM GN [11]. Deficiency of TNFR1 had no net effect on development of renal injury in this model. These studies are consistent with a major role for intrinsic renal cells as both the cellular source and target for TNF-mediated inflammatory injury in crescentic GN.

Studies in IL- $1 \beta$ chimeric mice demonstrated that protection from crescentic GN and functional renal injury in the absence of leukocyte-derived IL- $1 \beta$ was similar to the protection observed in mice totally deficient in IL-1 $\beta$ indicating a dominant role for leukocyte-derived IL-1 $\beta$. In the absence of intrinsic renal cell IL-1 $\beta$ production, crescent formation and injury were minimally affected although some reduction in glomerular recruitment of leukocytes $\left(\mathrm{CD} 4^{+} \mathrm{T}\right.$ cells and macrophage) was observed, suggesting a role for renal derived IL-1 $\beta$ in a feedback loop that augments renal leukocyte recruitment. In addition, glomerular TNF expression was significantly reduced in the absence of leukocyte IL-1 $\beta$ but unaffected by the absence of IL-1 $\beta$ production by intrinsic renal cells, suggesting a sequence of involvement of these cytokines in which leukocyte-derived IL- $1 \beta$ plays a major role in stimulating intrinsic renal cell TNF production resulting in crescentic injury.

The principal cellular targets for IL-1 $\beta$ in this model were studied in chimeric mice with selective expression of the signalling receptor for IL-1 $\beta$; the type I IL-1 receptor (IL-1RI) [12]. The type II IL-1 receptor is a non-signalling decoy receptor for IL-1. Absence of intrinsic renal cell IL-1RI provided similar protection to complete IL$1 \mathrm{RI}$ deficiency, indicating that intrinsic renal cells are the major targets for IL-1 $\beta$. In contrast, chimeric mice with intact renal expression of IL-1RI but absent bone marrow IL-1RI expression developed crescentic GN similar to normal mice. The effects of intrinsic renal cell IL-1RI deficiency on crescentic renal injury and glomerular TNF production were similar to those of leukocyte IL-1 $\beta$ deficiency providing further evidence for a dominant role of leukocyte-derived IL-1 $\beta$ acting principally on intrinsic renal cells (via IL-1RI) to induce TNF expression and crescentic injury.

\section{Intrinsic Renal Cell Production of IL-12 and IFN- $\gamma$}

Both IL-12 and IFN- $\gamma$ are important cytokines in generation of T-helper 1 (Th1) subset responses which are involved in cellular immunity and delayed-type hypersensitivity. IL-12 production by antigen-presenting cells (APCs) promotes Th1 differentiation and IFN- $\gamma$ production by $\mathrm{T}$ cells amplifies their IL-12 responses and activates macrophages. Both these cytokines have been dem- onstrated in crescentic forms of human GN. In experimental GN, their presence augments Th1 responses and crescentic injury.

IL-12 is produced by tubular epithelial cells and mesangial cells in vitro, but little was known about the contribution of renal production of this cytokine to GN. Using IL-12 chimeric mice, deficiency of renal IL-12 production was shown to attenuate development of crescentic GN without attenuation of the systemic immune response to the nephritogenic antigen [13]. This suggests a significant local pro-inflammatory contribution of IL-12 production by intrinsic renal cells to crescentic GN, in addition to the role of IL-12 in initiation and amplification of nephritogenic Th1 responses. It should be noted that these mice were deficient in the $\mathrm{p} 40$ subunit of IL-12 which is also a component of IL-23; therefore a potential contribution of IL-23 to the observed responses needs to be considered in these studies.

IFN- $\gamma$ is predominantly produced by activated T cells and natural killer cells. It augments Th1 responses by inducing IL-12 receptor expression on T cells and upregulates $\mathrm{MHC}$ expression on macrophages. Its renal expression has been detected in human and experimental GN, but little is known about its production by intrinsic renal cells. Using IFN- $\gamma$ chimeric mice, a significant contribution of IFN- $\gamma$ production both leukocytes and intrinsic renal cells was demonstrated in crescentic GN [1]. Up-regulation of renal MHC-II expression showed a greater dependence on intrinsic renal cell IFN- $\gamma$ production than leukocyte IFN- $\gamma$ production. IFN- $\gamma$ expression was identified on tubular cells, whilst $\mathrm{CD} 8^{+}$cells were the principal leukocyte source of IFN- $\gamma$ in nephritic kidneys. Although $\mathrm{CD}^{+}$cells in the spleen produced IFN- $\gamma$, production by $\mathrm{CD}^{+}$cells in the kidney could not be detected. This suggests a key role for leukocyte-derived IFN- $\gamma$ from secondary lymphoid organs in directing nephritogenic Th1 responses, but a dominant role for intrinsic renal cells as the major source of IFN- $\gamma$ in nephritic kidneys.

\section{Intrinsic Renal Cells in Antigen Presentation and Co-Stimulation}

In addition to their capacity to produce pro-inflammatory mediators, intrinsic renal cells have the ability to express MHC-II and co-stimulatory molecules, suggesting involvement in antigen presentation and $\mathrm{T}$-cell activation. Delayed-type hypersensitivity responses play a critical role in crescentic forms of GN, but the 
Fig. 1. Typical appearance of a crescentic glomerulus in a mouse with anti-GBM antibody-initiated GN.

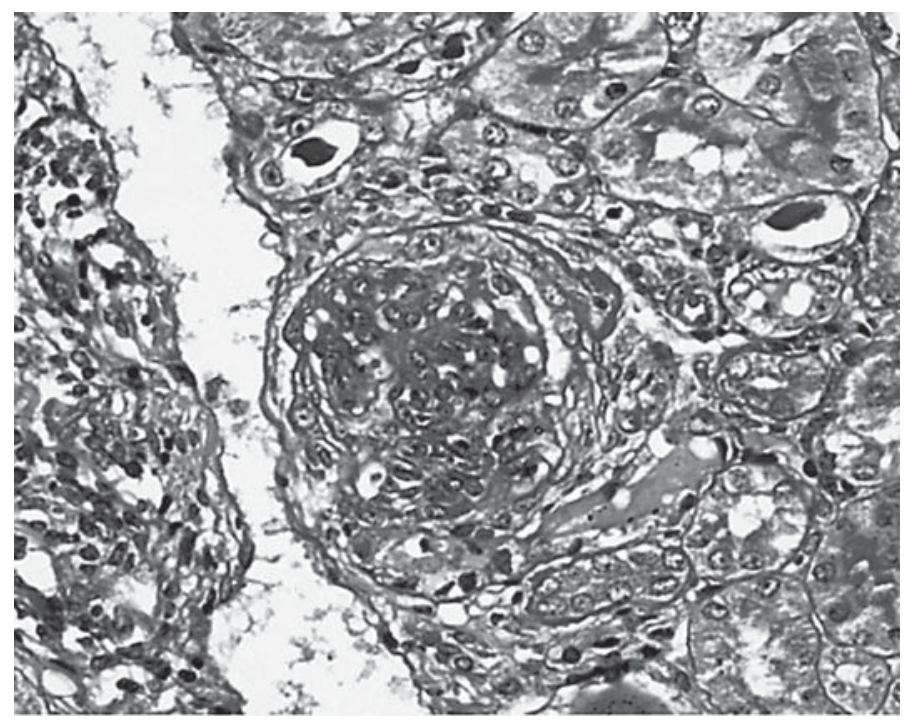

II-intact MRL/lpr mice developed GN and inflammatory cell infiltrates indicating that renal MHC-II is not required for immune complex-initiated renal injury.

Co-stimulatory molecules are expressed by professional APCs and their binding to specific receptors on T cells provides important second signals for activation of following TCR binding to MHC-II/peptide complexes. Signalling by co-stimulatory molecules is bi-directional and can enhance effector functions of macrophages and dendritic cells. CD40/CD154 is a co-stimulatory molecule pair expressed by APCs and T cells respectively, which in addition to enhancing $\mathrm{T}$-cell activation, also stimulates macrophage production of IL-12, IL-8 and IL-1 and upregulates MHC-II and ICAM-1 expression. CD40 expression is observed on mesangial cells, endothelial cells and tubular epithelial cells in patients with proliferative GN and is up-regulated on tubular epithelial cells in vitro by IL-1.

The contribution of CD40 on intrinsic renal cells to crescentic GN was studied using CD40 chimeric mice [16]. Mice with complete CD40 deficiency failed to develop a humoral or cellular immune response to the nephritogenic antigen and were protected from development of crescentic GN. Deficiency of CD40 expression on intrinsic renal cells did not affect the systemic immune response, but provided significant protection from development of crescentic GN. This was associated with reduced glomerular accumulation of $\mathrm{CD} 4^{+} \mathrm{T}$ cells and macrophages and reduced renal expression of chemokines, IP-10 and MCP-1. These studies demonstrate that although primary immune responses are intact in the ab- 
sence of renal CD40, their expression in the kidney during development of crescentic GN augments recruitment and activation effector cells and development of renal injury.

\section{Intrinsic Renal Cell Proliferation in Crescent Formation}

Crescents are a manifestation of the accumulation of cells, fibrin and matrix proteins in Bowman's space and result in compression of the glomerular tuft and obstruction to the flow of urinary ultrafiltrate (fig. 1). Crescent formation is a feature of severe forms of GN, which may have a rapidly progressive clinical course and are generally associated with poor outcome for renal function. The contributions of macrophage recruitment and proliferation of glomerular epithelial cells to the development of crescents has been the subject of debate over several decades. Studies in murine crescentic GN suggest that the majority of cells in crescents express ezrin, which is a marker of both visceral and parietal epithelial cells [17]. Many of these cells also express PCNA, indicating that they are actively proliferating.

Cre/ROSA26 transgenic mice, in which selective $\beta$-galactosidase expression in podocytes is driven by a Crepodocyte-specific promoter fragment, have provided a technique to detect the specific contribution of visceral epithelial cells (podocytes) to the development of crescents [18]. Approximately 25\% of cells in crescents expressed this podocyte marker, but interestingly other podocyte-specific markers such as WT-1, synaptopodin, nephrin and podocin could not be detected in these cells. Proliferation of both $\beta$-galactosidase-positive podocytes and $\beta$-galactosidase-negative cells was apparent in crescents.
Mice deficient in the cyclin-dependent kinase inhibitor, $\mathrm{p} 27^{\mathrm{Kip} 1}$, develop organomegaly and show enhanced cellular proliferation and crescent formation in GN [19]. Both humoral and cell-mediated immunity and the recruitment of leukocytes were unaffected in $\mathrm{p} 27^{\mathrm{Kip} 1}$. deficient mice. Transplantation of normal bone marrow into $\mathrm{p} 27^{\mathrm{Kip} 1}$-deficient mice did not affect the severity of crescentic GN, indicating that enhanced proliferation of intrinsic renal cells and not recruited leukocytes was responsible for the increased severity of GN resulting from excessive cellular proliferation caused by the absence of this cyclin-dependent kinase inhibitor in these mice.

\section{Conclusion}

The technique of bone marrow transplantation has proved a useful technique for dissecting the pro-inflammatory contribution of intrinsic renal cells and leukocytes in murine crescentic GN by allowing selective deletion of inflammatory molecules of interest from one of these cellular compartments. Studies have demonstrated important functional contributions of renal cell-derived IL-12 and INF- $\gamma$ and contrasting contributions of intrinsic renal cells and leukocytes with regard to production of TNF and IL-1 $\beta$. A role for MHC-II on intrinsic cells has been demonstrated in targeting T-cell-dependent immune effector responses to the kidney, and co-stimulatory molecules on intrinsic renal cells augment renal leukocyte recruitment and activation. Evidence is also accumulating that glomerular epithelial cell proliferation makes an important contribution to the cellular composition of crescents. There is considerable scope for studies using chimeric mice to reveal other pro-inflammatory roles of intrinsic renal cells in inflammatory models of renal injury.

\section{References}

$>_{1}$ Timoshanko JR, Holdsworth SR, Kitching AR, Tipping PG: IFN- $\gamma$ production by intrinsic renal cells and bone marrow-derived cells is required for full expression of crescentic glomerulonephritis in mice. J Immunol 2002;168: $4135-4141$.

- 2 Imasawa T, Utsunomiya Y, Kawamura T, Zhong Y, Nagasawa R, Okabe M, Maruyama N, Hosoya T, Ohno T: The potential of bone marrow-derived cells to differentiate to glomerular mesangial cells. J Am Soc Nephrol 2001;12:1401-1409.
3 Ito T, Suzuki A, Imai E, Okabe M, Hori M: Bone marrow is a reservoir of repopulating mesangial cells during glomerular remodeling. J Am Soc Nephrol 2001;12:2625-2635.

-4 Noronha IL, Eberlein-Gonska M, Hartley B, Stephens S, Cameron JS, Waldherr R: In situ expression of tumor necrosis factor- $\alpha$, interferon- $\gamma$, and interleukin-2 receptors in renal allograft biopsies. Transplantation 1992;54: 1017-1024.

\footnotetext{
5 Neale TJ, Ruger BM, Macaulay H, Dunbar PR, Hasan Q, Bourke A, Murray-McIntosh RP, Kitching AR: Tumor necrosis factor- $\alpha$ is expressed by glomerular visceral epithelial cells in human membranous nephropathy. Am J Pathol 1995; 146:1444-1454.

-6 Tesch GH, Yang N, Yu H, Lan HY, Foti R, Chadban SJ, Atkins RC, Nikolic-Paterson DJ: Intrinsic renal cells are the major source of interleukin- $1 \beta$ synthesis in normal and diseased rat kidney. Nephrol Dial Transplant 1997;12: 1109-1115.
} 
7 Yokoo T, Ohashi T, Utsunomiya Y, Shen JS, Hisada Y, Eto Y, Kawamura T, Hosoya T: Genetically modified bone marrow continuously supplies anti-inflammatory cells and suppresses renal injury in mouse Goodpasture syndrome. Blood 2001;98:57-64.

$>8$ Tipping PG, Leong TW, Holdsworth SR: Tumor necrosis factor production by glomerular macrophages in anti-glomerular basement membrane glomerulonephritis in rabbits. Lab Invest 1991;65:272-279.

$>9$ Tipping PG, Lowe MG, Holdsworth SR: Glomerular interleukin-1 production is dependent on macrophage infiltration in anti-GBM glomerulonephritis. Kidney Int 1991;39;103110.

${ }_{10}$ Timoshanko JR, Sedgwick JD, Holdsworth SR, Tipping PG: Intrinsic renal cells are the major source of tumor necrosis factor contributing to renal injury in murine crescentic glomerulonephritis. J Am Soc Nephrol 2003;14: 1785-1793.
1 Vielhauer V, Stavrakis G, Mayadas TN: Renal cell-expressed TNF receptor 2 , not receptor 1 , is essential for the development of glomerulonephritis. J Clin Invest 2005; 115:1199-1209.

12 Timoshanko JR, Kitching AR, Iwakura Y, Holdsworth SR, Tipping PG: Leukocyte-derived interleukin-1 $\beta$ interacts with renal interleukin-1 receptor I to promote renal tumor necrosis factor and glomerular injury in murine crescentic glomerulonephritis. Am J Pathol 2004;164:1967-1977.

13 Timoshanko JR, Kitching AR, Holdsworth SR, Tipping PG: Interleukin-12 from intrinsic cells is an effector of renal injury in crescentic glomerulonephritis. J Am Soc Nephrol 2001; 12:464-471.

14 Li S, Kurts C, Kontgen F, Holdsworth SR, Tipping PG: Major histocompatibility complex class II expression by intrinsic renal cells is required for crescentic glomerulonephritis. J Exp Med 1998;188:597-602.

15 Mukherjee R, Zhang Z, Zhong R, Yin ZQ, Roopenian DC, Jevnikar AM: Lupus nephritis in the absence of renal major histocompatibility complex class I and class II molecules. J Am Soc Nephrol 1996; 7:2445-2452.
16 Ruth AJ, Kitching AR, Semple TJ, Tipping PG, Holdsworth SR: Intrinsic renal cell expression of CD40 directs Th1 effectors inducing experimental crescentic glomerulonephritis. J Am Soc Nephrol 2003;14:2813-2822.

17 Ophascharoensuk V, Pippin JW, Gordon KL, Shankland SJ, Couser WG, Johnson RJ: Role of intrinsic renal cells versus infiltrating cells in glomerular crescent formation. Kidney Int 1998;54:416-425.

18 Moeller MJ, Soofi A, Hartmann I, Le Hir M, Wiggins R, Kriz W, Holzman LB: Podocytes populate cellular crescents in a murine model of inflammatory glomerulonephritis. J Am Soc Nephrol 2004; 15:61-67.

$>19$ Ophascharoensuk, V, Fero, ML, Hughes, J, Roberts, JM, Shankland, SJ. The cyclin-dependent kinase inhibitor p27Kip1 safeguards against inflammatory injury. Nat Med 1998;4, 575-580. 